CHERCHER, REPÉRER, AVANCER

\title{
LA « FORCE DE L'ÉVÉNEMENT » EST-ELLE UN ARTEFACT ?
}

Les mobilisations de victimes au prisme des théories événementielles de l'action collective

Stéphane Latté

Presses de Sciences Po (P.F.N.S.P.) | « Revue française de science politique »

2012/3 Vol. 62 | pages 409 à 432

ISSN 0035-2950

ISBN 9782724632590

Article disponible en ligne à l'adresse :

http://www.cairn.info/revue-francaise-de-science-politique-2012-3-page-409.htm

\section{Pour citer cet article :}

Stéphane Latté, «La « force de l'événement » est-elle un artefact ? Les mobilisations de victimes au prisme des théories événementielles de l'action collective », Revue française de science politique 2012/3 (Vol. 62), p. 409-432.

DOI 10.3917/rfsp.623.0409

Distribution électronique Cairn.info pour Presses de Sciences Po (P.F.N.S.P.).

(C) Presses de Sciences Po (P.F.N.S.P.). Tous droits réservés pour tous pays.

La reproduction ou représentation de cet article, notamment par photocopie, n'est autorisée que dans les limites des conditions générales d'utilisation du site ou, le cas échéant, des conditions générales de la licence souscrite par votre établissement. Toute autre reproduction ou représentation, en tout ou partie, sous quelque forme et de quelque manière que ce soit, est interdite sauf accord préalable et écrit de l'éditeur, en dehors des cas prévus par la législation en vigueur en France. Il est précisé que son stockage dans une base de données est également interdit. 


\section{LA «FORCE DE L'ÉVÉNEMENT" EST-ELLE UN ARTEFACT?}

AU PRISME DES THÉORIES ÉVÉNEMENTIELLES DE L'ACTION COLLECTIVE

\section{Stéphane Latté}

$\mathrm{D}$ ans les publications académiques comme dans les récits indigènes, l'émergence, depuis vingt-cinq ans, d'associations de « victimes »- sur le terrain des attentats, des accidents industriels ou des catastrophes naturelles - et la logique des engagements militants qui s'y rencontrent sont fréquemment rapportées à la confrontation à un événement inaugural (la mort d'un enfant, la destruction de l'environnement familier, l'expérience de la peur face à un accident hors du commun, etc.), aux conséquences traumatiques qui lui succèdent et aux bouleversements des schèmes de compréhension du monde qu'elle est supposée engendrer. L'événement dramatique se voit ainsi prêter une capacité à susciter lui-même des groupes mobilisés et à produire, du seul fait de sa force et de son évidence, des griefs et des mécontentements. Pour expliquer les mobilisations de "victimes ", la tentation est alors grande de faire régresser l'analyse vers cette boîte noire qu'est l'événement. Par son évidence, ce dernier façonnerait les unités pertinentes de mobilisation (des victimes se reconnaissant immédiatement comme telles). Il créerait automatiquement des solidarités entre ceux qui eurent l'infortune de le rencontrer. Il heurterait de façon telle ses victimes que ces dernières disposeraient directement de bonnes raisons d'agir collectivement.

Tout semble en fait incliner le chercheur à attribuer un rôle causal à l'événement dramatique. D'abord, parce que la plupart des associations de « victimes » naissent (chronologiquement) de l'irruption de l'événement fortuit dans le cours de la vie ordinaire. Ensuite, parce que l'identité collective revendiquée publiquement par ces groupes se fonde sur la mise en scène de solidarités d'accident qui paraissent s'originer dans le vécu commun d'un même drame, indépendamment des vecteurs ordinaires de la sociabilité militante. Enfin, parce que cette forme émergente d'action collective est socialement problématisée dans les termes de la rupture événementielle. Loin d'être un temps socialement suspendu, la situation de catastrophe est parcourue de normes qui règlent la manière dont les acteurs se perçoivent, s'expriment et se déplacent. Or, l'idée d'une détermination des acteurs par l'événement doit beaucoup à la manière dont le rôle de "victime » s'est institutionnalisé en Francel. Les savoirs et les dispositifs au carrefour desquels ce dernier s'est construit ont notamment contribué à durcir la frontière entre un avant et un après l'événement. Que l'on songe aux entrepreneurs de «victimologie » qui, autour du diagnostic de traumatisme psychique et des pratiques de

1. Nous avons retracé le processus d'institutionnalisation de la catégorie de "victimes " dans la thèse dont est issu cet article : Stéphane Latté, "Les “victimes”. La formation d'une catégorie sociale improbable et ses usages dans l'action collective», thèse pour le doctorat d'études politiques, Paris, EHESS, 2008. 
l'urgence psychologique, ont essaimé l'idée d'un rôle pathogène de l'événement ${ }^{1}$; à l'ingénierie commémorative dont la vocation consiste à produire des cérémonies qui célèbrent (et construisent) des communautés d'accident dépourvues d'autres attributs que celui d'un deuil partagé ${ }^{2}$; aux codes du journalisme de catastrophe qui réclament de ses témoins un récit biographique borné par les frontières temporelles de l'événement; ou encore aux pratiques d'expertise qui exigent des candidats à la réparation des dommages des narrations dans lesquelles les atteintes directement imputables à l'événement doivent être strictement dissociées des injustices antérieurement vécues.

Cette trame de la situation de catastrophe façonne donc les pratiques des victimes, mais elle gouverne également la focale à travers laquelle la sociologie les observe. C'est ce que nous souhaitons montrer dans cet article en nous fondant sur les matériaux d'une enquête consacrée aux usages de la catégorie de "victime » dans l'action collective - et plus particulièrement aux mobilisations consécutives à l'explosion de l'usine chimique AZF, à Toulouse en septembre $2001^{3}$. Dans quelle mesure la "force de l'événement » est-elle en effet autre chose qu'un artefact, tributaire à la fois du halo de préconstructions qui entoure les mobilisations dites de "victimes ", mais également du type de matériaux que recueille le chercheur sur ces terrains?

Nous reviendrons dans un premier temps sur la généalogie des outils conceptuels proposés, depuis 20 ans, par la sociologie des mobilisations pour isoler un "facteur événement » de l'action collective. Qu'il s'agisse d'invoquer un choc moral ${ }^{4}$, des griefs subitement imposés ${ }^{5}$ ou des contextes d'ébranlement $d u$ quotidien $^{6}$, la «force de l'événement» est le plus souvent présumée dès lors que les facteurs ordinaires de l'action collective paraissent mis en échec (un recrutement associatif inattendu, des alliances improbables, des carrières militantes "révélées » par la catastrophe). Sa présence est surtout attestée par un recours systématique aux témoignages recueillis auprès de «victimes".

Nous montrerons pourtant que le poids de la rupture événementielle ne saurait être authentifié en se fondant sur les seuls récits qu'en font les acteurs, en entretien ou sur la scène publique. Les narrations produites par les « victimes » sont en réalité largement tamisées par le régime de légitimation publique propre aux contextes de catastrophe et par les normes discursives (l'invocation, par exemple, d'un « choc») mises en circulation et surveillées par les psychologues, les journalistes et les entrepreneurs de mobilisation.

1. Stéphane Latté, Richard Rechtman, «Enquête sur les usages du traumatisme psychique », Politix, 73, 2006, p. 159-184.

2. Stéphane Latté, "Vous ne respectez pas les morts d'AZF". Ordonner les émotions en situation commémorative", dans Sandrine Lefranc, Lilian Mathieu (dir.), Mobilisations de victimes, Rennes, Presses Universitaires de Rennes, 2009, p. 205-220.

3. Ce texte s'appuie sur un corpus d'une cinquantaine d'entretiens réalisés auprès de dirigeants d'associations de notoriété nationale (Fédération nationale des victimes d'accidents collectifs, Association des parents d'enfants victimes, Fédération internationale des victimes de catastrophes aériennes, Union nationale des victimes de catastrophes) et auprès des acteurs engagés dans le mouvement "sinistré » qui a suivi l'explosion de I'usine chimique AZF. Ce dernier terrain a été principalement exploré à partir d'une enquête ethnographique menée au court de 5 séjours de 2001 à 2005. Les enquêtés et les lieux permettant de les identifier ont été anonymisés.

4. James M. Jasper, The Art of Moral Protest, Chicago, The University of Chicago Press, 1997.

5. Edward Walsh, "Resource Mobilization and Citizen Protest in Communities Around Three Mile Island ", Social Problems, 29 (1), 1981, p. 1-21.

6. David A. Snow, Daniel M. Cress, Liam Downey, Andrew W. Jones, «Disrupting the "Quotidian" : Reconceptualizing the Relationship Between Breakdown and the Emergence of Collective Action ", Mobilization, 3 (1), 1998, p. 1-22. 
Dès lors, nous militerons pour un changement de prisme méthodologique. Le recours à l'ethnographie permet en effet d'amender l'hypothèse discontinuiste et de réinscrire ces mobilisations « accidentelles » dans des histoires sociales au long cours : celle des dispositions et des engagements passés qui incline certains individus (mais pas tous) à endosser le rôle social de "victime"; celle des espaces militants qui conduit certains groupes (mais pas tous) à se convertir en " communauté d'accident ». Loin de dénier toute force à l'événement, cet article se propose au contraire d'analyser la manière dont les conjonctures dramatiques rebattent les cartes de la protestation en imposant de nouvelles règles à l'énonciation des griefs et en offrant de nouveaux cadres à la dénonciation de l'injustice.

\section{Des « victimes » mobilisées par l'événement ? Les hypothèses du groupe sans attache et du mécontentement nu}

$\mathrm{L}$ a sociologie des mobilisations de "victimes " s'est principalement construite autour de l'hypothèse du groupe sans attache. Dans cette perspective, certaines propriétés morphologiques paraissent distinguer les associations de « victimes » des autres formes de l'action collective. Cette spécificité réside principalement dans les effets fédérateurs qu'exerce l'événement dramatique et dans le peu de poids des supports ordinaires de mobilisation. Des individus dont le cours de la vie a été ébranlé par l'irruption de l'événement fortuit parviennent à se mobiliser sur le seul fondement du «mauvais hasard » qui les a réunis. Qu'on les nomme, avec Jean-Paul Vilain et Cyril Lemieux, des « groupes circonstanciels " ${ }^{1}$, ou, à la suite de Stefaan Walgrave et Joris Verhulst, des "nouveaux mouvements émotionnels $»^{2}$, ces collectifs ont pour propriété principale de reposer uniquement sur un lien accidentel. Des solidarités nées directement de l'événement parviennent à se substituer aux ressorts ordinaires de l'action collective: le partage d'affinités sociales ou politiques préalables, le soutien de réseaux d'interconnaissance ou le recours à des agences spécialisées de mobilisation. Dans une comparaison historique suggestive entre deux événements semblables (l'incendie d'un cinéma en 1947 et celui d'un institut thermal en 1991), Vilain et Lemieux dressent ainsi une opposition franche entre deux manières de produire du collectif à la suite d'un sinistre : la première, prévalente en 1947, est dite "catégorielle » car elle se fonde sur des « solidarités a priori », des réseaux fermement enracinés dans la vie quotidienne (syndicats, cercles de voisinage, clientèles politiques). La seconde - les « groupes circonstanciels »- repose au contraire sur des « solidarités a posteriori » directement organisées autour du vécu commun de l'événement. Les associations de victimes contemporaines s'apparenteraient de ce point de vue à des "groupes sociaux à l'état brut " dans la mesure où elles naissent d'une rencontre fortuite, d'une détermination purement événementielle, du seul fait de s'être retrouvés ensemble « au mauvais endroit, au mauvais moment ».

«Par l'intermédiaire de tels groupes, que I'on propose de qualifier de "circonstanciels", des personnes accèdent à une existence politique qui, d'une part, se passe du soutien des appareils de mobilisation traditionnels (partis politiques, syndicats, associations déjà instituées, etc.) et, d'autre

\footnotetext{
1. Jean-Paul Vilain, Cyril Lemieux, «La mobilisation des victimes d'accidents collectifs. Vers la notion de "groupe circonstanciel" ", Politix, 44, 1998, p. 135-160.

2. Stefaan Walgrave, Joris Verhulst, "Toward "New Emotional Movements" ? A Comparative Exploration into a Specific Movement Type », Social Movement Studies, 5 (3), 2006, p. 275-304.

3. Les deux auteurs demeurent toutefois prudents sur ce point en évoquant, en incise, la possibilité d'une relative homogénéité sociale du recrutement associatif (J.-P. Vilain, C. Lemieux, "La mobilisation des victimes d'accidents collectifs... », art. cité, p. 148.
} 
part, n'est plus directement référée à des appartenances sociales conventionnelles (professionnelles, religieuses, sexuelles, culturelles, locales, etc.). Dans ce nouveau contexte de l'action collective, les personnes engagées ne s'appuient tendanciellement que sur un seul point commun: elles ont toutes subi de plein fouet un même événement tragique qu'elles n'avaient en rien suscité ni recherché. II s'agit donc sous ce rapport d'une forme de mobilisation qui ne repose pratiquement, en amont, sur aucun socle institutionnel ou communautaire préalable, et ne débouche, en aval, sur aucune extension politique ou chaînage idéologique de grande ampleur, parce qu'elle est seulement fondée sur une condition de victime que l'État n'a pas su prévenir. ${ }^{1}$

Corollaire de l'hypothèse du groupe sans attache, l'hypothèse du mécontentement nu la complète et la prolonge. Car, outre sa capacité à façonner des groupes, l'événement peut se voir attribuer un pouvoir propre de création et d'ordonnancement des griefs. Par l'ampleur de leurs dommages et par la violence des sentiments qu'ils suscitent, les événements dramatiques généreraient un socle de souffrances partagées capables par elles-mêmes d'engendrer la mobilisation. Depuis vingt ans, de nombreux auteurs se sont ainsi efforcés de réintroduire un «facteur événement " traditionnellement délaissé par la sociologie de l'action collective. Dans un article séminal, Edward Walsh montre par exemple comment, en 1979, à la suite de l'accident nucléaire de Three Mile Island ${ }^{2}$, un tissu de petites communautés conservatrices, composées de classes moyennes faiblement disposées à l'action collective, est devenu, en quelques semaines, un foyer d'activisme, jusqu'à figurer parmi les symboles du mouvement antinucléaire américain. Walsh propose ainsi le concept de "griefs subitement imposés " (suddenly imposed grievances) pour qualifier ces situations exceptionnelles dont l'ampleur enjoint une part des individus qui les vivent à s'organiser collectivement (l'accident de Three Mile Island, mais également la marée noire de Santa-Barbara, la contamination chimique de Love Canal ou la catastrophe de Seveso).

De même, en se fondant notamment sur l'exemple de la mobilisation antinucléaire de Diablo Canyon qui capte des acteurs parfois éloignés de l'idéal-type de l'activiste (des fermiers et des mères au foyer des classes moyennes), James Jasper affirme qu'un danger, un accident ou la mort d'un enfant « suscitent chez la personne un sentiment d'outrage tel qu'elle devient encline à l'action politique, avec ou sans les réseaux interpersonnels qu'invoquent habituellement la théorie de la mobilisation des ressources $»^{3}$. Comme le résume Daniel Cefaï, «les "chocs moraux" produisent une prise de conscience brutale des problèmes et conduisent à s'engager pour une cause $»^{4}$. Sans être tout à fait automatiques ${ }^{5}$, ces indignations immédiates jouissent d'une efficacité proprement affective : "[Le terme] de "choc" renvoie davantage à la puissance émotionnelle de ces expériences. Puisque l'image sous-jacente est celle de l'état de choc ou du choc électrique, cela implique des sensations viscérales et corporelles, de même nature que les vertiges ou les nausées $»^{6}$.

1. J.-P. Vilain, C. Lemieux, ibid., p. 136.

2. E. Walsh, "Resource Mobilization... », art. cité.

3. J. M. Jasper, The Art of Moral Protest, op. cit., p. 106.

4. Daniel Cefaï, «Les cadres de l'action collective : définitions et problèmes ", dans Daniel Cefaï, Danny Trom (dir.), Les formes de I'action collective. Mobilisations dans des arènes publiques, Paris, Éditions de I'EHESS, 2001, p. 57-91, dont p. 73.

5. Pour James Jasper, la force mobilisatrice de l'événement reste en effet modulée, en amont, par les dispositions biographiques des individus (qui déterminent la « résonance symbolique » et la valeur accordée à l'objet menacé) et, en aval, par les efforts que produisent les entrepreneurs de mobilisation pour canaliser, orienter et convertir l'indignation immédiate en un sentiment d'outrage dirigé.

6. James M. Jasper, "The Emotions of Protest: Affective and Reactive Emotions in and around Social Movements ", Sociological Forum, 13 (3), 1998, p. 397-424, dont p. 409. 
On doit enfin à David Snow et à ses collaborateurs la dernière tentative d'intégration du facteur événementiel aux théories de l'action collective ${ }^{1}$. À rebours des extensions parfois données au concept de "choc moral », les auteurs s'efforcent de circonscrire le domaine de pertinence des causalités événementielles de l'action collective. Ils identifient des catégories d'événements au potentiel particulièrement mobilisateur, qu'ils définissent non pas par leurs propriétés morphologiques - leur soudaineté ou leur violence - mais par le type d'effets qu'ils produisent sur les individus qui les vivent. Les sociologues délimitent ainsi des contextes «d'ébranlement du quotidien " (quotidian disruption). Figurent notamment dans cette catégorie les quotidian disrupting accidents, des événements que distingue une faculté particulière à perturber les routines ordinaires, à briser les supports - y compris matériels - des croyances de la vie de tous les jours, à mettre en doute l'ordre naturel du monde. Ainsi, les victimes de catastrophes collectives ne seraient pas agies par un choc mécanique lié à la soudaineté de l'accident, mais par une disposition à vouloir restaurer l'ordre du quotidien. Se trouvent réunies sous ce concept toutes les situations qui occasionnent une "violation de l'environnement protecteur », qui s'introduisent dans ces zones qui, à l'instar du domicile ou de la famille, sont culturellement définies comme des espaces privatifs, normalement protégés des agressions du monde extérieur. Par exemple, les mères endeuillées de l'association américaine de victimes de la route Mothers against drunk driving (MADD), ainsi que la plupart des groupements NIMBY (Not in my backyard) cherchent-ils, après une infraction commise par un agent étranger, à "reconquérir leur zone de contrôle et à reconstituer leur bulle protectrice $»^{2}$.

Le principal apport de D. Snow et de ses collaborateurs tient en l'effort produit pour identifier les maillons de la séquence causale de l'(in)action collective que l'événement vient altérer. Plutôt que d'invoquer des causalités purement événementielles, les auteurs réexaminent l'ensemble des opérations de cadrage qui permettent d'ordinaire à l'action collective de se déployer, mais à l'aune, cette fois, des transformations qu'exercent les contextes d'ébranlement du quotidien. Ces conjonctures critiques ont notamment pour principal effet de renforcer le cadre motivationnel (les bonnes raisons d'agir et, plus précisément, d'agir collectivement) sans que des organisations n'aient à intervenir. L'événement modifie de ce fait la perception des coûts et des rétributions relatifs à l'action collective : "Quand le quotidien est ébranlé, nous pouvons présumer que les calculs qui conduisent à l'action ne sont plus les mêmes. À la différence des mouvements qui doivent motiver les individus pour compenser les risques associés à la participation, il est probable que la rupture du quotidien offre par elle-même une incitation suffisante à l'action ${ }^{3}$.

En dépit de leurs différences, ces entreprises de réhabilitation du rôle de l'événement se heurtent toutefois à des difficultés théoriques identiques. Le recours au schéma de la rupture événementielle explique en effet à la fois trop et trop peu. Trop, car dès lors qu'une mobilisation est constatée, l'explication par l'événement ne peut guère être démentie et elle réduit à une même cause l'ensemble des engagements disparates qui s'y agrègent ${ }^{4}$. Trop peu, car

1. D. A. Snow et al., "Disrupting the "Quotidian”... », art. cité.

2. D. A. Snow et al., ibid., p. 8.

3. D. A. Snow et al., ibid., p. 17.

4. Ainsi des mises en garde que formulent Francesca Polletta et Edwin Amenta contre un usage trop extensif de la notion de «choc moral »: "Virtuellement, tout événement ou toute nouvelle information peut rétrospectivement être qualifiée de choc moral. Dès lors, nous devons nous demander ce qui, dans certains événements, crée tant de colère, d'outrage et d'indignation que ceux qui s'y trouvent exposés sont conduits à protester. Existe-il une classe particulière de problèmes plus susceptibles que d'autres de créer des chocs moraux ? [...] 
la «force de l'événement» rend incompréhensible les situations d'apathie ${ }^{1}$. Pourquoi, confrontées à un même drame, certaines victimes, leur immense majorité sans doute, ne prennent-elles part à aucune action collective? Dans une nuance qui fragilise l'édifice qu'ils viennent de construire, D. Snow et son équipe doivent ainsi convenir que les mêmes causes événementielles ne produisent pas toujours les mêmes effets de mobilisation.

«Il y a évidemment des exemples de ruptures du quotidien, comme le cas de certains désastres ou déplacements de populations, qui fragilisent les liens sociaux existants, qui démoralisent ceux qu'elles affectent et qui finalement rendent improbable une action collective quelle qu'elle soit, et plus particulièrement une mobilisation soutenue. Cette remarque suggère qu'il existe un seuil au-delà duquel l'ébranlement du quotidien et l'action collective deviennent antinomiques. » ${ }^{2}$

Mais, au-delà de ces difficultés, les théories événementielles de l'action collective s'exposent surtout à un problème méthodologique car, faute d'une prise en compte suffisante des motifs obligés qui encadrent le discours des acteurs en contexte de catastrophe, elles enregistrent des ruptures que les enquêtés sont mis en demeure d'attester.

\section{Quand le discours « personnel ॥ devient une parole institutionnelle : I'inversion des règles du travail de représentation}

$\mathrm{L}$

'hypothèse du groupe sans attache et celle du mécontentement nu paraissent, en première analyse, particulièrement adéquates à la description des mobilisations de "victimes ». Ces interprétations trouvent en tout cas une série d'échos et de confirmations dans le matériel recueilli sur nos terrains d'enquête. Le récit des origines qu'offrent, par exemple, les dirigeants du mouvement des sinistrés de l'explosion de l'usine AZF combine ainsi l'essentiel des motifs qui supportent l'interprétation événementielle des mobilisations post-catastrophiques. Ainsi de la plaquette de présentation publique de cette association de sinistrés toulousaine :

«Ça s'est fait au hasard de la rencontre de deux voisins qui, sans se connaître, furent réduits par la même explosion au triste statut de sinistrés. Quelques affichettes très artisanales, apposées sur les platanes du quartier, invitant les habitants à une réunion publique sur le parking de la piscine, ont suffi. Ils sont venus. Avec leur colère, leur désolation, leur solitude et leurs pansements. Les initiateurs, debout sur deux chaises - forcément bancales - tentent, à l'aide d'un mégaphone

\footnotetext{
Spécifier quand les chocs moraux ont des chances de se produire apparaît crucial. Autrement, nous risquons de parvenir à la même circularité que celle de certains arguments de la théorie du processus politique: tout ce qui précède une protestation devient une opportunité politique ou, ici, un choc moral » (Francesca Polletta, Edwin Amenta, "Second that Emotion? Lessons from Once-Novel Concepts in Social Movement Research", dans Jeff Goodwin, James M. Jasper, Francesca Polletta (eds), Passionate Politics. Emotions and Social Movements, Chicago, The University of Chicago Press, 2001, p. 303-316, dont p. 307-308).

1. Ruud Koopmans et Jan Duyvendak évoquent ainsi les failles du concept de "griefs subitement imposés " qui, selon eux, "présuppose ce qui devrait en réalité faire l'objet de l'explication»: "Labelliser les accidents nucléaires comme des "griefs subitement imposés", c'est [...] ne se concentrer que sur les accidents qui ont effectivement conduit à la mobilisation d'activistes (soit les cas où le grief objectif et le grief subjectivement ressenti coïncident), en mettant de côté le fait que la plupart des incidents nucléaires n'ont provoqué que de faibles, voire d'inexistantes protestations (certains ont même pu ne jamais parvenir jusqu'à nous). Comme le démontre le cas de Tchernobyl, le même accident devient un événement politique majeur dans un pays tandis qu'il ne suscite dans les autres pas plus de controverses qu'un bulletin météo » (Ruud Koopmans, Jan W. Duyvendak, "The Political Construction of the Nuclear Energy Issue and its Impact on the Mobilization of AntiNuclear Movements in Western Europe ", Social Problems, 42 (2), 1995, p. 235-251, dont p. 248).
}

2. D. A. Snow et al., «Disrupting the "Quotidian"...", art. cité, p. 19. 
récupéré à la hâte, de répercuter, d'organiser et de solliciter la parole. Elle fuse. Beaucoup de colère, immédiate, incontrôlable, comme l'événement. Chacun amène ses doléances et son courroux, sa solitude et sa peur. Tous expriment une colère qui ressemble de très près à celle du désespoir. Cet étrange rassemblement, entre cour des miracles et meeting revendicatif, va trouver son unité, son identité, dans l'exigence, unanime et récurrente de voir la justice passer. C'était le 23 septembre 2001.»

Ce tropisme de l'événement, le poids des émotions violentes qu'il a pu engendrer, l'intensité du « choc » qu'il a provoqué, l'impériosité des motivations à agir qu'il a pu susciter, parcourent également nombre des entretiens que nous avons menés auprès de dirigeants d'associations de «victimes ». Quel statut dès lors attribuer à ces émotions «brutes ", «livrées » en entretien, à ces récits de malheur, à ces chroniques détaillées du déroulé du drame, à ces confessions d'expériences traumatisantes dans lesquelles semblent s'originer l'action collective? S'agit-il de les enregistrer comme preuves de la manifestation d'un «choc moral» («Rien ne sera jamais plus comme avant») ou faut-il les réduire à de simples scories du discours? Convient-il d'en faire l'armature de l'explication ou, au contraire, de les remiser telle une forme locale de "langue de bois » ? Ces questions ne sont pas sans créer un certain inconfort. Invoquer des contextes narratifs, des stratégies de légitimation, des modes de présentation de soi routinisés risquerait de paraitre dénier, en la mettant à distance, la réalité des souffrances effectivement vécues et exprimées, de révoquer ces moments denses qu'expérimentent en entretien les enquêtés, mais aussi l'enquêteur. A contrario, la prendre à l'état de donnée, d'expression nue, supposerait de renoncer aux règles élémentaires de contrôle des matériaux, de s'épargner l'analyse du statut à attribuer à la parole recueillie. Il n'est pas donc question ici de dénier la force de l'événement, mais d'évaluer sa place dans le récit.

Le vécu du drame personnel peut en effet être livré, confessé, raconté, mais aussi résumé, éludé, escamoté, ou tout simplement tu. Or, parmi les manières de dire l'événement, le corpus de nos entretiens fait apparaître des variations significatives et, en un sens, contreintuitives. Les règles ordinaires de la pudeur qui interdisent généralement de se confier à un étranger paraissent en effet déroutées. Comme le rappelle Olivier Schwartz, " compte tenu du rapport privé/public tel qu'il fonctionne dans les sociétés occidentales, il est peu probable que les régions les plus intimes du privé [puissent] accéder à l'expression en dehors d'une communication elle-même privée, supposant le secret et donc l'absence du magnétophone ${ }^{1}$. S'agissant d'individus revêtus de l'habit de représentant associatif, s'ajoutent généralement aux codes de la discrétion les contraintes de désingularisation qui astreignent le discours à s'abstraire du cas personnel ${ }^{2}$. Or, au cours de l'enquête, ces deux prérequis apparurent suspendus, si ce n'est inversés. Plus nous entretenions un rapport de familiarité et une relation durable avec nos enquêtés, plus les marques de l'événement semblaient s'effacer, au point de parfois devenir invisibles. En compulsant nos retranscriptions après-coup, nous pouvions nous apercevoir que nous ignorions souvent le détail des dommages personnels subis par nos interlocuteurs les plus proches. De même, plus nous retardions le moment de l'entretien dans le parcours d'enquête, plus le propos se délestait du récit subjectif de la catastrophe. Paradoxalement encore, le degré d'intimité du témoignage semblait comme augmenté par les réticences et la méfiance manifestées par l'enquêté lors de la prise de contact. Ce technicien

1. Olivier Schwartz, Le monde privé des ouvriers, Paris, PUF, 1990, p. 48.

2. Luc Boltanski, Yann Darré, Marie-Ange Schiltz, "La dénonciation », Actes de la recherche en sciences sociales, 51,1984, p. 3-40. 
de l'usine AZF se montra par exemple rétif à accueillir un sociologue qu'il estimait appartenir aux groupes professionnels (journalistes et enseignants) les plus réfractaires à l'industrie chimique. Or, durant l'entretien finalement timidement concédé, il se protégera de toute intrusion sur des sujets devenus localement problématiques (les conditions de travail à l'usine qu'incrimine l'enquête judiciaire, les actions syndicales brocardées dans la presse, l'entente des salariés avec leur direction que stigmatisent les mobilisations adverses), en orientant systématiquement la conversation vers l'évocation subjective du sinistre.

La place de la confession dans la chronologie de l'interaction interroge également puisqu'il s'agit le plus souvent d'un préalable posé par l'enquêté et non d'une "vérité » patiemment accouchée par l'enquêteur, au terme d'une mise en confiance propice à la confidence. Ainsi, plus nous nous adressions à des acteurs investis d'un mandat - présidents d'associations de notoriété nationale notamment -, plus ces derniers étaient familiers de la parole publique, plus l'irruption de l'événement dans la vie personnelle se situait en préambule de la discussion. Ici, le « personnel » devient le quasi-synonyme « d'institutionnel ». Les « régions intimes du vécu » deviennent les plus officielles. Par exemple de ce dirigeant d'un important regroupement de victimes de catastrophes aériennes, par ailleurs directeur de recherche en biologie, qui bouscule d'emblée la distance ordinaire de l'interlocution :

"Stéphane. On se tutoie parce que, vous savez, on va parler de choses tellement personnelles, que je ne peux pas vous appeler "monsieur", même si je ne vous connais pas. »"

Alors même que nous n'avions eu le temps de poser la moindre question, il engage, pendant près d'une heure, un récit heurté de l'accident qui l'endeuilla treize ans auparavant:

«Écoutez, il faut que je vous prévienne de quelque chose. Ce n'est pas la première fois que je parle de mon parcours, de ma vie. J'ai même la tendance inverse. Depuis que cet accident a eu lieu, quand je fais une nouvelle rencontre, j'ai tendance à commencer par ça. Tôt ou tard, je l'introduis parce que je trouve que ça a tellement ébranlé ma vie que... je me sens pas obligé, mais ça vient tout seul parce que ça fait partie de moi et je veux être pris aussi avec ça. J'ai beaucoup eu l'occasion d'en parler... c'est pas la première fois... j'ai travaillé tout ça depuis plusieurs années. Mais, malgré tout, il y a quand même des fois où l'émotion vient, donc vous m'excuserez si parfois... $»^{2}$

Confession sans confesseur, maïeutique qui se passe d'obstétrique, anamnèse qui parfois soliloque, l'expression de l'outrage privé se donne alors à voir comme une convenance publique. Non que cette parole soit «convenue » - artificielle, factice ou stratégique - mais simplement qu'elle n'est pas jugée "inconvenante", ou mieux, qu'il convient de la dire publiquement. Ainsi peut-on parler d'anonymat du témoignage en nom propre.

\section{Marché du témoignage et circulation des récits traumatiques}

e discours sur l'événement doit en fait être ressaisi dans son contexte d'énonciation et mis en relation avec les normes qui l'encadrent. D'abord, en rappelant qu'il a fait l'objet d'un apprentissage préalable acquis, pour les dirigeants associatifs, dans la répétition des interviews journalistiques et, parfois, dans cette pratique singulière de publicisation

1. Entretien, biologiste, président d'une fédération de victimes d'accidents aériens, mars 2005.

2. Ibid. 
des causes qu'est l'écriture autobiographique, ou, pour les victimes ordinaires, dans la fréquentation des cellules d'urgence, des expertises, des consultations psychologiques, des récits médiatiques également. En effet, autour des accidents collectifs se déploie désormais un dispositif serré de recueil de la parole intime sur le drame. Depuis la mise en place en 1997 de "SAMU Psy » dans chaque département, le recours aux cellules de soutien psychologique est devenu un instrument routinier de la communication publique en contexte de crise. Elles figurent aujourd'hui parmi les techniques ordinaires de démonstration du pouvoir politique dans des situations catastrophiques où l'effectivité de ce dernier devient problématique. À Toulouse, à la suite de l'explosion de l'usine AZF, ce sont par exemple plus de 450 thérapeutes qui, à l'appel du maire de la ville, se disperseront sur les territoires sinistrés. Cette diffusion d'une problématisation psychologique de la catastrophe est par ailleurs soutenue par les marchés privés et humanitaires de l'aide d'urgence. Tandis que chaque organisation caritative nationale (Croix-Rouge, Secours catholique, Médecins du monde) dépêche son propre staff de cliniciens, de nombreux employeurs (EDF, Sanofi, Total, DDE) délèguent également l'activité de soutien de leurs salariés à des prestataires extérieurs qui mettent à leur disposition soit des plateformes téléphoniques pour écouter les plaintes, soit des groupes de parole et des consultations individuelles. Enfin, ce marché des récits traumatiques a la particularité d'avoir été nourri aussi bien par des services publics de la santé ou par des officines privées que par des syndicats et des groupements de défense de victimes. On rappelle en effet que le « choc traumatique » est l'une des rares catégories psychologiques d'interprétation du malheur à avoir été revendiquées par ses malades. Tant en France qu'aux États-Unis, des mouvements sociaux - à l'instar de SOS-Attentats - ont ainsi directement concouru à l'élaboration et à l'officialisation du diagnostic de traumatisme psychique ${ }^{1}$. Depuis, le traumatisme intègre la boîte à outils discursifs dans laquelle puisent régulièrement les associations de victimes pour fédérer des groupes en construction qu'aucune histoire commune ne vient cimenter.

$\mathrm{Au}$ croisement de ces initiatives se met donc en place un réseau particulièrement dense de diffusion des modèles de mise en forme de l'événement et le langage du psychotraumatisme fait l'objet de larges campagnes de publicisation par le biais de fiches d'information distribuées dans les quartiers ou de placards affichés dans les cages d'escalier (et parfois traduits en arabe). Bien sûr, cette trame narrative peut être refusée $e^{2}$, tordue (lorsqu'au sein de l'usine AZF, le traumatisme vient qualifier la fermeture administrative de l'usine plutôt que l'explosion) ou stratégiquement appropriée (ce militant sinistré qui affirme que «le fric pour le traumatisme, ça a été une rétribution de mon activisme syndical $»^{3}$ ). Mais, dès lors que la conformité au récit traumatique devient une condition de la reconnaissance collective comme de l'allocation individuelle de ressources (une majorité des victimes d'AZF seront indemnisées

1. Sur la participation des mouvements sociaux à la réforme du diagnostic, voir : Wilbur J. Scott, "Post-Traumatic Stress Disorder in DSM-III. A Case in the Politics of Diagnosis and Disease ", Social Problems, 37 (3), 1990, p. 294-310; Allan Young, The Harmony of Illusions Inventing Post-Traumatic Stress Disorder, Princeton, Princeton University Press, 1995 ; S. Latté, R. Rechtmann, "Enquête sur les usages du traumatisme psychique ", art. cité.

2. Ainsi de ce militant d'extrême gauche grièvement blessé au cours de l'explosion, qui interpelle, à l'occasion d'un colloque, les professionnels de santé présents dans la salle: "Les cellules d'aide psychologique ont été notre premier contact avec les autorités sanitaires. J'ai moi-même été reçu par une dame qui m'a conseillé de faire un travail sur moi, parce que "la colère est mauvaise conseillère" ; moi, je crois au contraire qu'en pareilles circonstances, ce qui aurait été anormal, c'est de ne pas être en colère et je pense même que la reconstruction passe par cette colère qu'il faut transformer en lutte que j'espère collective " (Intervention lors du colloque organisé par I'Institut de veille sanitaire: "Surveillance sanitaire après une catastrophe. Que nous a appris I'explosion de l'usine AZF ? », Toulouse, 20 octobre 2006).

3. Entretien, infirmier, militant syndical à SUD, septembre 2004. 
au titre du " traumatisme »), les argumentaires idoines peuvent être plus ou moins fidèlement reproduits, sans que l'on puisse postuler ni une adéquation à l'expérience vécue, ni une intériorisation subie de modèles de narration prescrits. Ainsi cette accueillante d'une association de sinistrés de l'explosion d'AZF évoque-t-elle l'adhésion des habitants de son quartier à la mise en récit d'un événement systématiquement rapporté à un "choc».

«Les gens savent comment présenter les choses. [...] Si t'as pas été blessé, c'est tout de suite le "choc". [...] Les gens des quartiers, ils vont pas te dire: "C'est le préjudice moral". Ça, ils savent pas ce que c'est, il faut quand même avoir un petit peu réfléchi au problème pour prétendre au préjudice moral, c'est abstrait, donc ils te disent: "le choc, voilà on est très choqués". Donc le "choc", ils savent ce que c'est. $Y$ en a un qui est allé à l'expertise, il a vu qu'on lui parlait de ça et donc il l'a dit, puis le voisin l'a dit. »'

Une autre scène put nous interpeller dans le même sens. Une représentante associative, dure de caractère et peu prompte dans le quotidien du travail militant à l'épanchement intime, nous surprit en accordant à un journaliste de télévision, devant la façade du local associatif, un témoignage "sur mesure ", au sens où son récit, focalisé sur le souvenir de l'explosion, fut conclu d'un sanglot étranglé. Sa fille, spectatrice, nous glissa dans un sourire : "Elle sait faire ». Or, s'il n'est pas permis de douter de la sincérité des larmes, force est de constater que l'interaction fonctionne en premier lieu parce qu'un «savoir-faire », ici un sens pratique préréflexif, saisit la variabilité des frontières du public et du privé, qui ne sont pas les mêmes qu'en contexte ordinaire. La façade proposée dans le rôle d'accueillant - la mise à distance de son cas personnel - s'effondre, ou plus précisément se transforme, est remplacée par une autre, tout aussi sincère, tout aussi composée.

Le sociologue se trouve donc inclus, souvent malgré lui, dans un réseau plus large de sollicitation de l'évocation intime de l'événement qui pèse sur la nature du propos recueilli ${ }^{2}$. Nous intervenions de ce fait sur le marché saturé des récits de catastrophe, que d'autres acteurs - journalistes et psychologues notamment - avaient façonné et réglementé avant que nous n'y pénétrions. Certes, le choix, dans la présentation de l'enquête, du rattachement disciplinaire à la "science politique " put parfois se révéler précieux pour démanteler le malentendu qu'entretient volontiers la perception ordinaire entre sociologie et psychologie, entre une discipline mal identifiée et une autre devenue familière dans le contexte de catastrophe, pour mettre à distance les modes de narration que l'on sait pertinente pour la seconde (le « choc » ou le «traumatisme »). Mais, s'il y a "plusieurs agents dans une vie et plusieurs histoires de vie possibles pour chaque agent $»^{3}$, la seule mention de l'objet de l'enquête suffisait généralement à prédéfinir le rôle social à endosser et à favoriser l'adoption des trajets et des séquences typifiées qui lui correspondent. L'enquêté se sait interrogé comme "victime» ou comme représentant d'une "association de victimes». Il ne parlera donc pas comme l'enseignant, le père de famille, le fils de réfugié espagnol, le militant de la LCR, l'habitant d'un quartier populaire, qu'il est aussi mais «par ailleurs ». L'itinéraire est écrit

1. Entretien, employée, accueillante salariée dans une association de sinistrés, septembre 2004.

2. Sur ces questions, voir Pierre Bourdieu, «L'illusion biographique", Actes de la recherche en sciences sociales, 62 (1), 1986, p. 69-72. Pour une tentative de resituer l'entretien sociologique dans le spectre des dispositifs qui participent de "l'institutionnalisation du moi », voir Bernard Pudal, « Du biographique entre "science" et "fiction". Quelques remarques programmatiques", Politix, 27, 1994, p. 5-24. Pour une réflexion du même ordre dans le cas spécifique des contextes de catastrophe, voir Violaine Girard, Julien Langumier, "Risque et catastrophe. De I'enquête de terrain à la construction d'objet ", Genèses, 63, 2006, p. 128-142.

3. Yves Clot, «L'autre illusion biographique », Enquête, 5, 1989, 〈http://enquete.revues.org/document99.html〉. 
par avance, puisque s'invite clandestinement dans le dialogue une grille souterraine d'entretien, définie non par l'enquêteur, mais par l'enquêté, et en tête de laquelle figure cette question qu'il n'est pas nécessaire de poser : «Que s'est-il passé pour vous ce jour-là? » Ainsi, «commencer par le commencement » est-ce toujours faire état de l'événement inaugural (interrogeant des militants associatifs, d'autres points de départ étaient pourtant envisageables, la généalogie des engagements civiques, par exemple). Si bien que, lorsque, pour contourner l'écran de la rupture événementielle, nous tentions d'introduire l'entretien en sollicitant un «parcours », ce dernier se confondait en fait pour l'interlocuteur avec sa trajectoire de « victime».

Cette hypertrophie de l'événement se traduit enfin par une série de malaises. Gêne d'abord dans l'interaction quand nous étions mis en demeure d'écouter ce que nous ne nous sentions pas habilité à entendre. Ainsi de cette représentante de commerce qui, croisée quelques jours après une entrevue avec son époux, nous remercie : "Ça lui a fait du bien de parler avec toi de l'explosion, il en parle jamais à la maison, ça l'a fait avancer ». De même de cette secrétaire médicale qui nous incite à fixer un entretien avec son fils : «Je pense que ça pourrait l'aider ». Gêne ensuite dans la conduite de l'interaction car, comme le signalent avec justesse Julien Langumier et Violaine Girard, "l'omniprésence de la catastrophe détourne l'entretien ethnographique de sa visée classique : le récit du vécu accapare le récit de vie ${ }^{1}$. Plus précisément, l'empreinte de l'événement appose sur le discours des surimpressions, des éclairages, des trop pleins, des éléments projetés, et donc immanquablement - en miroir - des silences, des creux, des censures, des ombres portées, des angles morts. Difficile en effet, dans ce cadre, de solliciter un passé antérieur à l'événement qui, certes, pourrait éclairer les logiques d'engagement victimaires à l'aune des critères classiques de la sociologie des mobilisations, mais qui, dans ce contexte de sollicitation, peut devenir mal à propos ou simplement hors champ. En entretien, les trajectoires professionnelles, les solidarités préalables, les affiliations politiques et les adhésions associatives préexistantes disparaissent pour devenir opaques, voire, dans certains cas, indicibles dès lors qu'il est socialement admis (requis) que la vérité des victimes et de leur mobilisation se concentre tout entière dans l'événement.

\section{La fabrication médiatique des communautés d'accident}

$\mathrm{L}$ 'écran de l'événement s'insinue donc dans les matériaux produits par le chercheur. Il s'immisce également dans les sources de presse qui, du fait des préconstructions qui les soutiennent, confortent et accréditent à leur tour une lecture événementielle des engagements victimaires. Forme d'action collective aujourd'hui dotée d'une certaine historicité, "l'association de victimes" est prise dans des conventions héritées qui règlent la manière dont les groupes et leurs porte-parole se donnent à voir dans la presse. Or, ces dernières risquent d'amener à confondre le groupe tel qu'il est et tel qu'il se (qu'on le) présente et à sous-estimer ainsi le poids du substrat social sur lequel il s'appuie. De ce fait, la rencontre fortuite de "mauvais hasard " relève pour une part d'un mode typifié de présentation de soi. Puisque le partage d'affections conjoncturelles fonde l'identité publique de ces mouvements, les dirigeants associatifs doivent opérer tout un travail de dénégation des affinités politiques et des attributs sociaux préalables. "On ne se connaissait pas avant», « on s'est rencontré par hasard » : ces affirmations peuvent bien n'être que des constats, mais

1. V. Girard, J. Langumier, "Risque et catastrophe... », art. cité, p. 130. 
dès lors qu'elles sont estimées comme devant figurer dans le discours public, elles deviennent aussi des étendards. Il s'agit donc de les confronter à l'observation des canaux effectifs du recrutement associatif.

Sur le terrain de l'explosion de l'usine AZF, l'événement et le contexte de crise n'impulsent en réalité pas de renouvellement radical du recrutement militant. Après le 21 septembre, les variables « lourdes » de l'engagement demeurent robustes, l'appartenance à une communauté de destin n'abrase qu'à la marge le jeu des déterminants classiques de l'action collective et les carrières militantes "révélées » par l'événement demeurent exceptionnelles. Les dispositions sociales à l'engagement et les savoir-faire militants acquis préalablement conservent toute leur efficace. Ainsi, la population des militants sinistrés recouvre en large part celle des adhérents multicauses qui nourrissent, depuis bien des années souvent, les mouvements sociaux toulousains. Les réseaux militants préconstitués et les agences traditionnelles de protestation - organisations de la gauche mouvementiste (G10-Solidaires, SUD, FSU, DAL), partis politiques d'extrême gauche (LCR, Motivé-e-s) ou associations écologistes (Amis de la terre) - façonnent ainsi, dès les premières heures, le militantisme sinistré toulousain. En important dans la mobilisation les cadres d'injustice hérités de leurs combats antérieurs, ces organisations prendront une large part du travail d'imputation des griefs et ils offriront un soutien logistique essentiel aux manifestations de rue inaugurales qui appellent, à partir du 23 septembre, à la fermeture de l'usine chimique et à la réparation des dommages. Comme le relève Franck Weed à propos de la militance américaine des victimes de la route, nous sommes moins souvent en présence de «victimes devenues activistes » que «d'activistes confrontés à l'expérience de la victimation " ${ }^{1}$. L'événement dramatique oriente l'affectation des placements militants, plus qu'il ne les suscite.

Pourtant, des exigences conjoncturelles de présentation publique des groupes tendent à jeter dans l'ombre le passé militant des « sinistrés » mobilisés. Le contexte de catastrophe dans lequel l'action collective se déroule modifie en effet la hiérarchie relative des ressources à faire valoir et il redistribue les droits à la parole publique. Ainsi, à la suite du 21 septembre, l'arène médiatique gagne en centralité. Or, les règles et les normes qui y ont cours participent de la mise en scène de victimes profanes tout entières définies par le drame. Se mobiliser en tant que victime d'un événement dramatique suppose dès lors de renoncer, au moins provisoirement, toujours partiellement, en tout cas publiquement, à d'autres traits saillants de l'identité sociale, comme l'appartenance politique. Cette ressource circonstancielle qu'est l'expérience directe de la catastrophe se trouve monétisée, assurant de ce fait la visibilité nouvelle de profanes (des «victimes») et obligeant au retrait (du moins public) de nombreux « militants».

Les canons du journalisme de catastrophe accordent en effet une place centrale au format du témoignage individuel de victime uniquement qualifiée par le dommage subi. S'accumulent ainsi dans la presse les pastilles illustratives en forme de biographies affectées et les portraits de victimes ordinaires aux prises avec l'événement extraordinaire. Dès lors, bien qu'omniprésents dans le mouvement sinistré, les " encartés ", ceux dont l'appartenance politique ou syndicale est de notoriété publique, savent que les attributs attachés au rôle médiatique de victime risque de disqualifier par avance leur discours. Les activistes multicauses peuvent certes être aussi, mais par ailleurs, des sinistrés et attester de dommages personnels. Mais, ils n'en resteront pas moins associés publiquement à leurs engagements antérieurs, au 
point de figurer dans la presse au titre de "soutiens » ou de "sympathisants " plutôt que de "victimes ». Les militants de SUD ou de la LCR, qui peuplent la cause sinistrée à Toulouse, doivent ainsi s'ajuster à une réforme conjoncturelle des titres à parler qu'ils s'efforcent de devancer. Aussi renvoient-ils préférentiellement les journalistes vers des partenaires dont la vocation semble être née ex abrupto au lendemain de l'explosion, des anonymes qui cessent rapidement de l'être, ou qui ne l'étaient déjà plus tout à fait, mais qui ne portent pas avec eux le soupçon de l'antériorité politique. Ce syndicaliste, cheville ouvrière d'un collectif associatif de sinistrés, indique par exemple :

«Avant, on était toujours demandeurs, [les journalistes] nous voyaient arriver sur la Palestine, sur les sans-papiers, sur le chômage, sur les retraites, ils en avaient marre de voir toujours les mêmes tronches. Donc avec l'explosion, ils avaient besoin de têtes nouvelles, de mecs qui étaient sinistrés, mais qu'ils n'avaient jamais entendus. »"

Le dirigeant d'une autre association de sinistrés précise dans le même sens :

«Dans l'association, il y a des gens déterminés, mais qui sont souvent coincés par un mandat ou un engagement politique. Ils sont repérés comme étant militants politiques. Les actifs, Patrick, Thibault, Catherine, tous ont un engagement. Donc, il a fallu sortir quelqu'un du chapeau. C'est ce qui faisait ma pertinence, entre guillemets, je n'avais aucune étiquette assignable. Moi, j'aimerais bien qu'on puisse mettre un mec de SUD et de la Ligue. Dire qu'on n'est pas que sinistré, qu'on est sinistré, mais aussi chasseur, pêcheur, militant, philatéliste. $»^{2}$

Les rares militants de longue date à survivre à ce renouvellement conjoncturel des porte-parole sont ceux dont le stigmate laissé par l'explosion est suffisamment visible et sans appel pour pouvoir gommer l'antériorité politique et corroborer ainsi le schème journalistique de la rupture événementielle. Par exemple, ce membre de Lutte ouvrière, promu parmi les leaders sinistrés, constate-t-il avec ironie que la balafre qui lui court de l'œil jusqu'à la nuque lui a valu de bénéficier "d'un sésame qui [lui] a ouvert la porte des médias » : "Ça m’a donné une légitimité de vrai sinistré $»^{3}$. Et d'ajouter, dans un sourire: "Ils ont quand même pas eu de bol, le seul mec blessé de la cité, c'était aussi le seul militant». Les notices biographiques rapportées dans les médias nationaux ignorent ainsi le plus souvent ses antécédents militants, le patronyme disparaît derrière le seul prénom et la qualification par le dommage prime sur l'étiquette partisane : « Jean-Michel, blessé de la cité Bellefeuille ». Lorsque exceptionnellement l'attribut politique est signalé, toujours en passant, une description fournie des atteintes personnelles le précède.

«À côté, dans la cité Bellefeuille, qui jouxte le Mirail, Jean-Michel Godart, professeur à I'IUFM, était assis à sa table de travail: "La porte-fenêtre m'a balancé dans l'escalier. Je me suis relevé. Mon visage et mon cou saignaient fort. Tous ceux qui me voyaient s'évanouissaient". II est défiguré, sa carotide touchée. II perdra 3 litres de sang et ne devra sa survie qu'à un extraordinaire sang-froid. [...] Dimanche 21 octobre, cité Bellefeuille, Jean-Michel Godart est rentré. Une Iongue cicatrice barre son visage et son cou. Militant de Lutte ouvrière, il vit dans la cité. Enfin, il vivait, car son bâtiment, le plus touché, a été évacué. Le lotissement est petit, tout le monde se connaît. »

\footnotetext{
1. Entretien, ingénieur dans une entreprise publique, militant syndical à SUD et membre fondateur d'une organisation de sinistrés, avril 2002.

2. Entretien, travailleur social, fondateur d'une association de sinistrés, septembre 2005.

3. Entretien, enseignant, militant à Lutte ouvrière et dirigeant d'un collectif de sinistrés, septembre 2002.

4. L'Express, 15 novembre 2001.
} 
Les journalistes locaux, pourtant familiers du personnage pour l'avoir fréquenté lors de mobilisations antérieures (il fut tête de liste aux élections régionales de 1998 et bat régulièrement campagne pour les mouvements de sans-papiers), escamotent pour leur part le détail biographique dissonant, préférant insister sur une bifurcation biographique imputée au drame. Ainsi, de ce rédacteur de la presse régionale :

« Jean-Michel, c'est compliqué. Son origine politique, ça me dérange pas de l'écrire, mais jusqu'à un certain point, si c'est un papier politique par exemple [...]. Mais, il a été touché physiquement, donc pour nous, pour les médias, Jean-Michel, c'est d'abord le type qui a échappé à la mort. Avec son histoire, on peut faire un superlatif magnifique. C'est ça sa vraie légitimité pour la plupart des médias. Quelque part, il est là pour ça. Si je voulais être cynique, je dirais que c'est son utilité médiatique. [...] Moi, je sais que sa légitimité, elle vient aussi de tout le boulot qu'il a fait avant au nom de son engagement politique. II a choisi d'habiter là, dans cette cité, il a choisi de structurer une résistance à ce qu'il considère comme devant être combattu, avant l'explosion, pendant l'explosion et après l'explosion. »'

Cette mise en conformité avec le modèle accidentel vaut pour les biographies individuelles aussi bien que pour les présentations de soi collectives. Si cet enquêté se trouve dépouillé, en partie malgré lui, du travail militant vieux de plus de 20 ans qui nourrit son action au lendemain de l'explosion, il concourt également - par le travail de représentation qu'il met en œuvre après le 21 septembre - à la production du prototype médiatique de l'association de « victimes » strictement définie par l'événement subi. «Établi » pour des motifs politiques dans une cité populaire voisine d'AZF, ce militant trotskiste initie dans son bloc HLM une mobilisation pour la réparation des dommages qui ne dépareille pas des actions de politisation «par le bas» menées par le « comité de résidents de la cité Bellefeuille » qu'il anime depuis 1986. Or, l'anticipation des attentes médiatiques le conduit, après deux mois de relative invisibilité publique, à convertir ce "comité des résidents » doté localement d'une forte identité sociale en un "Collectif des sans-fenêtres", un label qui élime les propriétés sociales du groupe mobilisé, pour ne plus faire apparaitre que la seule qualification par les dommages de l'événement. La trouvaille sémantique vaudra à son auteur une amplification médiatique remarquable puisque le label intègre rapidement le langage commun du sinistre AZF, en s'autonomisant du groupe qu'il qualifiait originellement. Un journaliste du Monde saluera par exemple l'invention « d'une nouvelle catégorie de handicapés sociaux : les "sansfenêtres" $»^{2}$, tandis que Libération consacrera un billet au succès du "vocable de "sansfenêtres" désignant nouvellement les victimes collatérales du big bang d'AZF ${ }^{3}$.

Les exigences de la bonne présentation médiatique en contexte de catastrophe conduisent donc à la réduction des trajectoires individuelles à la seule confrontation à l'événement, à la transformation de groupes réels en "groupes circonstanciels", à l'effacement des «solidarités a priori » au profit de la manifestation exclusive de solidarités d'accident, à la disparition des liens sociaux enracinés derrière l'image d'une rencontre purement événementielle. Elles conduisent ainsi à l'hypostase d'une population - «les victimes d'AZF » - dotée de réactions et de propriétés propres, isolée des autres groupes sociaux, composée d'individus détachés de leurs attributs ordinaires.

\footnotetext{
1. Entretien, journaliste dans un hebdomadaire local, septembre 2002.

2. «À Toulouse, les sans-fenêtres se mobilisent», Le Monde, 13 novembre 2001.

3. "Les fenêtres et la porte", Libération, 13 novembre 2001.
} 


\section{Le décloisonnement en acte du rôle de « victime » : sur quelques bénéfices de la méthode ethnographique}

$\mathrm{P}$ our réinscrire l'événement dans des temporalités plus amples, pour saisir le statut de «victime» dans ses articulations aux rôles et aux appartenances sociales de la vie ordinaire, et pour « banaliser » l'analyse des engagements victimaires ${ }^{1}$, il convient donc d'agir, par le choix des méthodes, sur la nature des cadres d'énonciation et sur les conditions du recueil de ces "paroles de victimes ». À l'entame de notre enquête consacrée aux associations de "victimes", nous avions procédé à un premier balayage des organisations que nous estimions significatives de cette forme d'action (Fédération nationale des victimes d'accidents collectifs, Association des parents d'enfants victimes, Entraide de la catastrophe des hauteurs du Sainte-Odile, Association des familles de l'incendie Édouard Pailleron). Opérant par entretiens ponctuels, isolés les uns des autres, détachés des contextes de sociabilité, les matériaux se révélaient parfois décevants, car nous nous exposions au risque de produire ces « victimes » que finalement nous recherchions, soit des individus abstraits uniquement définis par l'événement subi, des êtres fictifs prédécoupés par le chercheur, des tranches de vie artificiellement arrachées des chairs de l'acteur social concret, des protagonistes unidimensionnels (des victimes sollicitées comme telles) et des trajets unidirectionnels (qui partent de l'événement - un accident ferroviaire, un incendie, un meurtre - pour aboutir à la mobilisation).

C'est donc pour contourner ce biais que nous avons choisi de resserrer la focale sur une situation particulière, celle des mobilisations consécutives à l'explosion de l'usine AZF survenue à Toulouse le 21 septembre 2001, et de la travailler à partir des principes de la méthode ethnographique.

L'implantation continue sur un terrain localisé permet ainsi d'appréhender les acteurs dans l'imbrication des différentes sphères qui composent leur vie sociale et à l'intersection des rôles qu'ils endossent successivement ou concomitamment. Comme l'indiquent Stéphane Beaud et Michel Pialoux, "par les multiples facettes d'un même personnage social qu'elle donne à voir, à des moments et dans des contextes chaque fois différents, la pratique régulière, sinon intensive, du "terrain" facilite la rupture avec la vision monolithique des mondes sociaux $»^{2}$. Olivier Schwartz évoque dans le même sens les vertus de la transversalité du regard ethnographique qui, en chassant hors du périmètre de recherche préconstruit en bibliothèque, permet d'appréhender ces «faits d'intersection » que sont en fait toujours les objets durcis du monde social : la méthode «met en garde contre la constitution d'unités isolées et monofonctionnelles $»^{3}$. L'insertion ethnographique se révéla de ce point de vue utile à la désubstantialisation en acte du rôle de "victime ». Nous avons en effet accepté l'offre qui nous a été faite de loger au domicile de nos enquêtés les plus proches (une famille implantée dans le quartier populaire du Mirail dont les trois membres animaient les permanences d'une association de sinistrés). Or, le partage des routines de ce foyer, de ses temps de loisir et non plus uniquement de ses activités comme militants sinistrés, contribua nettement au décentrement de notre regard. Le désenclavement du rôle

1. Nous suivons ici l'appel à la «banalisation» de l'analyse des mobilisations de victimes formulé par Sandrine Lefranc et Lilian Mathieu (Mobilisations de victimes, op. cit.).

2. Stéphane Beaud, Michel Pialoux, Retour sur la condition ouvrière, Paris, Fayard, 1999, p. 432.

3. Olivier Schwartz, "L'empirisme irréductible", postface à Nels Anderson, Le Hobo. Sociologie du sans-abri, Paris, Nathan, 1993, p. 265-308, dont p. 294. 
de " victime » ne correspondait plus à une pétition de principe, mais à l'expérience pratique vécue sur le terrain. En effet, tandis qu'initialement, nos journées étaient tout entières tournées vers l'évocation du sinistre AZF (au rythme des entretiens, des séquences d'observation dans le local associatif et des temps consacrés à la transcription), leur centre de gravité se déplaçait vers d'autres lieux (les espaces de la vie quotidienne) et sur d'autres sujets (les discussions anecdotiques, lors des repas familiaux ou des soirées entre voisins). Ces «victimes d'AZF » que nous avions rencontrées lors de nos entretiens préparatoires redevenaient, dans leur fréquentation journalière, ce qu'elles n'avaient jamais cessé d'être, soit des acteurs concrets, dont l'implication dans le drame demeurait une composante seconde de l'identité sociale. Dans les interstices et les relâchements qu'autorise l'insertion prolongée, le temps des acteurs sociaux ne se contracte plus autour de la seule explosion, les trajectoires se diluent et se décentrent, les éléments biographiques hors champ refont surface. Ainsi, pour les acteurs enquêtés par ce biais, l'engagement dans la cause sinistrée n'apparaît plus comme une réaction mécanique à un dommage hors du commun, mais prend sens dans la rencontre entre une offre conjoncturelle d'engagement et la succession des positions occupées dans le quartier, dans l'espace associatif local, sur le marché du travail, dans le ménage ou la fratrie (voir encadré ci-dessous). De même, dans la routine du travail associatif, les relations ne se donnent plus à voir sous le jour exclusif d'une solidarité automatique entre "victimes d'AZF", mais comme un jeu continu de froissements ou de convergences de dispositions : celles d'une secrétaire médicale et d'un cadre hospitalier ; d'un travailleur social syndiqué et d'un petit commerçant ; d'un militant de la LCR et de la femme d'un directeur d'usine, etc.

Le décentrement du regard qu'autorise la méthode ethnographique est également précieux sur ce terrain inconfortable qu'est le deuil. Certains de nos enquêtés ont en effet perdu un proche dans les événements que l'on prend pour objet. Or, la confrontation aux individus endeuillés s'accompagne d'un halo de timidités et d'inhibitions culturellement définies auxquelles l'acteur social qu'est le sociologue échappe difficilement. L'expérience du deuil écrase, souvent malgré eux, l'identité pour autrui qui s'impose aux membres d'associations de "victimes " et elle altère le cours de la relation d'entretien. Une "gêne " peut ainsi se manifester à chaque étape de l'enquête sociologique. Par une timidité d'abord dans les prises de contact, car le caractère accessoire de la requête peut sembler inconvenant au regard du sérieux des atteintes (lors de deux demandes d'entretien, les individus sollicités purent ainsi nous éconduire en nous rappelant l'inutilité de notre démarche et la perte de temps qu'elle constituait pour eux, compte tenu de l'ampleur du drame vécu). Par une autocensure, ensuite, dans la conduite de l'entretien (la difficulté à couper les récits affectés ou à poser des questions qui risqueraient de paraître déplacées). Par les réticences, enfin, à mobiliser dans l'analyse des schèmes explicatifs - les stratégies, les intérêts, les ressources, les dispositions, etc. - qui, par leur pouvoir d'objectivation, abrasent l'intensité subjective de l'expérience endeuillée. Or, la familiarité acquise dans la participation au quotidien d'une association contribue à lever cet embarras parce que, d'une part, l'enquêté ne s'exprime plus uniquement comme victime de la perte d'un proche (mais aussi, par exemple, comme un militant pris dans des rivalités et dans des stratégies) et que, d'autre part, la perception que s'en fait l'enquêteur se normalise et s'enrichit de nouveaux traits biographiques.

Le dernier ressort de la méthode ethnographique réside dans l'opportunité qu'elle offre de faire varier les cadres d'énonciation et de multiplier ce qu'Olivier Schwartz appelle des 
«situations de parole $»^{1}$. Ce que permet l'observation participante, ce n'est pas l'enregistrement d'une parole véridique qui serait libérée des biais introduits par cet artifice qu'est l'entretien. Toute scène d'expression (l'entretien comme les autres) impose des contraintes spécifiques qui tamisent et façonnent les narrations produites. En revanche, l'observation d'un même acteur dans des situations sociales différentes - une discussion de couloir, un entretien, une commémoration, une interview journalistique - permet de mesurer des écarts dans les postures et les discours. Or, ces variations invitent à réviser la place à accorder aux émotions suscitées par l'événement dans l'explication de la mobilisation car, dès lors que leur expression se transforme d'une situation à l'autre, il devient impossible de les rapporter à la seule objectivité des atteintes ${ }^{2}$. Par leur comparaison, ces contextes d'interlocution donnent ainsi à voir la manière dont les contraintes de rôle agissent sur l'énonciation des motifs, plutôt que les motifs eux-mêmes.

\section{Réinscrire les engagements victimaires dans des trajectoires sociales au long cours}

Pour illustrer la nécessité de rendre aux engagements victimaires leur inscription dans des trajectoires sociales au long cours qui ne se réduisent pas à l'événement subi, nous évoquerons le parcours de la secrétaire générale d'une des principales associations de sinistrés toulousaines. Élevée dans les milieux aisés et politisés de l'anarcho-syndicalisme catalan, cette enquêtée a fait l'expérience d'un déclassement social précoce, entamé par l'exil contraint de ses parents sous le régime franquiste, puis prolongé par une série d'accidents biographiques, dont son mariage avec un homme qui, pendant vingt ans, projeta le foyer dans un cycle de violences familiales. Locataire d'une cité HLM contiguë de l'usine AZF au moment de l'explosion, elle a fait l'essentiel de sa carrière au bas de la hiérarchie hospitalière comme agent de services en charge des ménages. Génératrice d'un habitus clivé, sa trajectoire se caractérise donc par une tension constante entre une identité sociale perçue (une appartenance au monde des lettrés) et des propriétés objectives qui résistent. Or, depuis sa retraite, elle aspire à s'extraire de sa condition sociale et résidentielle, en multipliant notamment les engagements associatifs dans les domaines de l'aide aux femmes battues et de l'éducation populaire.

La rencontre de cette enquêtée avec la cause des sinistrés se comprend d'abord au regard de ses expériences passées. Dans son évocation de la journée de l'explosion, s'entremêlent ainsi la peur immédiatement ressentie et le souvenir vivace de ses «années noires». Absente du quartier le 21 septembre tandis que sa famille logeait à moins d'un kilomètre de l'usine, elle rapporte son désarroi à la réminiscence des culpabilités rentrées qu'elle pouvait éprouver lorsqu'elle constatait son impuissance à extraire ses enfants d'un foyer menaçant. Ce décentrement par rapport à l'événement $A Z F$ se repère également dans les détournements du langage du traumatisme qu'opère cette actrice, dans son travail d'accueillante. Lorsqu'elle oriente ses adhérents vers les psychologues dépêchés après le 21 septembre, elle les invite systématiquement à « raconter leur histoire », à faire mention des périodes de chômage, des misères matérielles, de l'isolement du quartier, de leurs répercussions sur la cellule familiale. Et si les cliniciens tentent de ramener leurs interlocuteurs à un récit centré sur l'explosion, la militante les réoriente sans cesse vers l'expression des maux et des griefs passés. Elle conclura ainsi un debriefing en interpellant la thérapeute : « J'espère que vous noterez que le traumatisme, il était déjà présent avant l'explosion ».

1. O. Schwartz, ibid., p. 268.

2. Sur l'influence qu'exerce le dispositif commémoratif sur la manifestation publique des émotions, voir S. Latté,

" "Vous ne respectez pas les morts d'AZF"... », cité. 
Sa fréquentation de la militance de l'aide aux femmes battues a par ailleurs favorisé l'acquisition de savoir-faire et de grilles de déchiffrement directement transposables au lendemain de la catastrophe: une familiarité avec l'étiquette de « victime »; une connaissance des pièges et des ressources qu'elle recèle; une capacité à lier des drames privés à des causalités structurelles (qu'il s'agisse du patriarcat dans le cas des violences conjugales, ou du capitalisme dans celui d'AZF); une valorisation du témoignage public et de l'imputation judiciaire de responsabilités comme mode de réparation des injustices vécues; une théorie relativement explicitée des mécanismes de domination (de l'occultation, de l'acceptation, de la résignation, de l'incapacité à agir et à dénoncer); une dextérité également dans la prise de parole publique et plus spécifiquement dans cette forme particulière d'intervention politique qu'est l'expression de souffrances personnelles à articuler à des revendications de portée générale (l'enquêtée fait notamment valoir sa fréquentation antérieure des émissions de témoignage sur le sujet des violences faites aux femmes).

Mais l'adhésion à une association de sinistrés doit aussi aux qualités prêtées à ce type de mobilisation. Le monde social des «victimes » est réputé plastique, non que les hiérarchisations sociales s'évanouissent sous l'effet mécanique de solidarités d'accident, mais parce que l'illusion d'une communauté de destin vient (provisoirement, inégalement, toujours imparfaitement) les recouvrir. L'identité sociale paraît dans les premières semaines suspendue, elle demeure en tout cas silencieuse dans le quotidien associatif, au profit d'une identification circonstancielle fondée sur la proximité au drame. Il s'agit ainsi d'un espace ouvert, où seule semble autoriser à parler et à intervenir la réalité des dommages personnels et non plus les titres, les mandats, les qualités statutaires ou professionnelles. Pour une actrice traversée par une contradiction constante entre la classe objective et l'appartenance subjective, la militance victimaire s'offrira de ce fait comme un interstice où peuvent s'épanouir, dans l'oubli de la position sociale effective, des aspirations contrariées. Cette enquêtée transporte ainsi dans son engagement victimaire les logiques qui régissent également la sélection de ses amitiés, soit l'accès à des strates que sa profession et son lieu d'habitation interdisent ordinairement.

Enfin, a contrario des secteurs militants établis dont les positions de représentation paraissent encombrées par les adhérents de longue date, la militance victimaire offre, en raison du privilège médiatique accordé à la figure de la victime profane saisie par l'événement, un terrain favorable aux vocations tardives à l'action collective.

\section{Quand les supports traditionnels d'identification résistent à l'événement : de la labellisation refusée à l'importation sous contrainte d'une forme d'action}

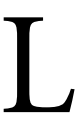

a méthode ethnographique opère en dernier lieu " comme un instrument de vigilance qui met en garde contre la tentation permanente de réification des groupes sociaux $»^{1}$. Ici, en adoptant une approche localisée, le groupe abstrait et homogène que semblent constituer les « victimes d'AZF » éclate en une multiplicité d'unités socialement pertinentes, dont la plupart se fondent sur les appartenances sociales conventionnelles et qui nourrissent, après le 21 septembre, le militantisme post-AZF : les «sinistrés du quartier Papus», les 
« résidents » de la cité Bellefeuille, les «travailleurs d'AZF », les « locataires de Jeambart », les " habitants de la rue Bernadette ", les « copropriétaires des Oustalous ", les commerçants du quartier Croix-de-Pierre, etc. Certains de ces groupes s'engageront dans un travail de conversion publique en communautés d'accident (par la création d'associations ad hoc empruntant le lexique de l'événement - "victimes ", " sinistrés ", "sans fenêtres ", "blessés ", etc.) quand d'autres continueront de se mobiliser sur le fondement de supports identitaires prééxistants. Dès lors, la recomposition des identités publiques après le 21 septembre ne saurait être uniformément imputée à la «force de l'événement ». Elle doit s'analyser comme le produit de la perception différenciée des atteintes et comme l'effet de l'inégale capacité des groupes à s'ajuster aux nouveaux cadres de la légitimation publique qu'impose la conjoncture dramatique. En atteste, notamment, les cas où les appellations circonstancielles ne se substituent aux formes «catégorielles " d'identification (la profession, la classe, l'appartenance syndicale, etc.) qu'au prix d'une importation sous contrainte.

Ainsi du mouvement des «locataires de la cité Jeambart » qui, durant l'année 2002, évolue en périphérie des principales associations de sinistrés pour exiger une réparation accrue des dommages. Dans la pétition qu'adressent les habitants de cet ensemble HLM, l'appartenance sociale, l'affiliation territoriale et le statut patrimonial l'emportent sur les identités publiques promues après l'explosion. La mention « victimes de la catastrophe du 21 septembre » n'est adjointe que tardivement, sur les conseils du dirigeant d'une association extérieure au quartier. Cette continuité dans les façons de se nommer prolonge une indifférenciation dans la perception des atteintes. Ici, la structuration du récit autour d'une partition nette entre un avant et un après AZF (celle-là même qui dirige la réparation assurantielle des dommages ou la présentation journalistique des témoignages) se révèle problématique, car les maux imputables à l'explosion peinent à être dissociés des difficultés antérieures que l'accident confirme, avive ou ratifie, plus qu'il ne les produit. Le sinistre s'insère en fait dans une temporalité plus ample qui embrasse un déclin, individuel et collectif, au long cours. L'isolement vécu dans la suite immédiate de l'explosion rappelle en effet l'enclavement progressif de la cité sur le territoire local ; la destruction matérielle des équipements collectifs participe d'une dégradation continue du cadre de vie engendrée par le déclin des investissements publics; l'exode de nombreux locataires au lendemain du 21 septembre fait écho au repli démographique du quartier et aux vagues successives de départs qui ont fragilisé les solidarités de voisinage ; la désorganisation dans la distribution des dons rappelle les concurrences ordinairement perçues sur le marché de l'aide sociale; les peurs mal contenues des enfants fragilisent un cadre éducatif qu'on peinait préalablement à maintenir, etc. Dès lors, des froissements se font jour entre le modèle accidentel de présentation des griefs qui prévaut sur les scènes locales de la réparation et l'expérience des atteintes vécues par ces acteurs. Que les initiateurs de ce mouvement sollicitent la médiation du maire, le concours d'une association de sinistrés ou le règlement du litige par la direction de l'office HLM, ils se voient systématiquement opposer la même fin de non-recevoir : "Vous mélangez tout ».

La mobilisation de «travailleurs d'AZF » illustre également la pluralité des supports d'identification mobilisables en contexte de catastrophe qui, tous, ne se fondent pas sur ce lien d'occasion qu'est le partage d'une même expérience dramatique. Cette communauté professionnelle a certes concentré une large part des dommages puisque 21 des 31 personnes décédées travaillaient sur le site et que l'explosion conduit à la disparition définitive de cet ancien bastion industriel, vieux localement de près d'un siècle. Pourtant, les affiliations professionnelles - l'appartenance au groupe ouvrier et l'exercice d'un métier industriel - vont 
durablement résister à l'offre de nouvelles identités circonstancielles, au point que les clivages consécutifs au 21 septembre se disent publiquement dans les termes d'une opposition entre "salariés » et "sinistrés ». Tandis que les militants associatifs scandent dans les manifestations "Salariés, sinistrés, solidarité », un large calicot barre pendant quelques semaines l'entrée de l'usine AZF : "Les salariés, solidaires des sinistrés ». Les pancartes des cortèges manifestants annoncent encore les "travailleurs d'AZF », alors que les titres de la presse confortent l'imperméabilité des deux groupes: "Salariés et victimes, toujours partagés ${ }^{1}$. De fait, chez la plupart des familles de salariés d'AZF décédés, l'attachement historique à l'entreprise, la transmission intergénérationnelle de l'attachement à l'usine, les réseaux d'amitié noués dans le travail, la reconnaissance à l'égard de la sollicitude patronale, la fidélité à une activité dont les proches continuent parfois de vivre, obstruent toute tentative de conversion à la cause des «victimes ». Rejetant les offres d'adhésion émises par les associations créées après l'explosion, elles maintiendront leur allégeance aux structures d'encadrement du groupe professionnel du défunt (à l'intersyndicale de l'usine, puis à l'association de mémoire des anciens travailleurs d'AZF).

De multiples facteurs pourraient être invoqués pour expliquer l'enracinement durable de ce clivage entre "victimes » et "salariés » et l'impossibilité de faire advenir une communauté d'accident unifiée. Il conviendrait bien sûr de rappeler la contradiction des intérêts matériels immédiats puisque, lorsque les collectifs associatifs font leur la revendication de fermeture définitive du pôle chimique, les salariés d'AZF exigent son redémarrage et le maintien d'une industrie locale intra-muros. Il faudrait encore évoquer la marginalisation progressive du groupe professionnel sur le territoire sinistré. Si, jusqu'aux années 1980, la cité ouvrière construite par l'entreprise chimique innervait les sociabilités de voisinage, les salariés du site ont progressivement déserté les quartiers attenant à l'usine pour accéder à la propriété en dehors de la ville. Riverains et salariés ne partagent désormais plus ni la même expérience résidentielle, ni les mêmes structures d'encadrement. On pourrait surtout rappeler les obstacles, désormais bien renseignés, qui interdisent l'endossement du rôle de " victime » lorsque l'événement est désigné comme un accident du travail ${ }^{2}$ (l'incorporation de la confrontation au danger comme expérience "normale », les stratégies professionnelles de mise à distance des situations d'accident ${ }^{3}$, les freins économiques et juridiques à la dénonciation publique du risque industriel par ceux-là même qui le subissent, etc.).

Toutefois, pour expliquer le maintien des supports d'identification préalables à l'explosion, l'invocation d'un groupe professionnel toujours identique à lui-même et d'une culture ouvrière immuable dans ses vertus et dans sa cohésion ne saurait suffire. La perpétuation des identités conventionnelles n'est guère plus mécanique que ne l'est la constitution de communautés d'accident. Elle procède en effet d'un travail a posteriori de réactivation et de réenchantement des «solidarités a priori ». De concert avec l'intersyndicale, la direction de l'usine AZF mène ainsi une politique interne qui vise à prévenir les risques d'adhésion à des identifications concurrentes ( sinistrés ", "victimes ", etc.). Dès les premiers jours, une vie d'usine se reconstitue sur les ruines d'un site pourtant dépourvu de toute activité productive.

1. La Dépêche du Midi, 12 mai 2006.

2. Emmanuel Henry, Amiante: un scandale improbable. Sociologie d'un problème public, Rennes, Presses Universitaires de Rennes, 2007 ; Jean-Noël Jouzel, «Encombrantes victimes. Pourquoi les maladies professionnelles restent-elles socialement invisibles en France ? "Sociologie du travail, 51 (3), 2009, p. 402-418.

3. Denis Duclos, "La construction sociale du risque. Le cas des ouvriers de la chimie face aux dangers industriels ", Revue française de sociologie, 28 (1), 1987, p. 17-42. 
En dépit de la raréfaction progressive du travail, la direction s'affaire à maintenir les temps sociaux et les supports matériels qui fondent la sociabilité d'usine. Un "village » s'organise autour d'un chapiteau dressé pour accueillir la cantine et un parterre de bungalows abrite les permanences syndicales, les services sociaux et les équipes de psychologues embauchés pour l'occasion. Pendant près de six mois, les solidarités d'atelier se perpétuent autour des anciennes lignes de production désaffectées et près de la moitié des salariés se rendent quotidiennement à leur poste. Les meetings organisés chaque semaine par le directeur de l'usine, les assemblées générales de salariés, les tournées syndicales nourrissent un tissu dense de relations sociales. Or, c'est dans ce huis clos que s'élabore et se solidifie un récit alternatif du drame dans lequel les salariés du site sont certes bien désignés comme victimes, mais victimes en premier lieu de la réprobation sociale qui vise leur industrie plutôt que de l'explosion.

Surtout, cette opposition des identités (re)mobilisée à la suite de l'explosion ne se comprend que si l'on envisage l'inégal ajustement des groupes mobilisés aux cadres d'énonciation de l'injustice qu'impose le contexte de catastrophe. La promotion du registre de la victimisation et le déplacement de la lutte vers les arènes médiatiques contraignent les délégués de l'usine à jouer "à l'extérieur », sur un terrain qui leur est étranger. L'agilité des représentants de sinistrés à manier le témoignage individuel contraste avec les réticences et la gêne qu'éprouvent, face à l'exercice, les syndicalistes de l'usine AZF. Conviés à s'exprimer dans le registre de l'émotion et de la peine, ces derniers refusent, pendant près de trois mois, d'accorder le moindre portrait individuel à la presse et préfèrent s'en tenir aux formes éprouvées de la communication syndicale - le communiqué de presse ou le cortège manifestant - que les journalistes estiment inadaptées aux exigences conjoncturelles du traitement du désastre.

«Moi, j'aurais aimé faire le portrait d'un salarié qui avait tout perdu ou dont le meilleur copain était mort ou qui avait passé toute la journée à sauver les copains, parce qu'il y a eu des actes héroïques, des salariés qui ont littéralement sauvé des collègues. [...] Ça, ça aurait été hyperfort aussi sur le plan journalistique. Mais personne n'était candidat à témoigner. Donc c'était tout le problème des journalistes: vous avez plein de gens qui veulent témoigner d'un côté, et aucun de l'autre. »'

En dépit des rappels réguliers à l'ordre de la catastrophe (« Vous êtes aussi des sinistrés, vous devez le montrer aux Toulousains $»^{2}$ ), témoigner en nom propre demeure perçu par ces représentants syndicaux comme une trahison à l'égard du mandat que l'on a reçu des collègues, une réticence au récit de soi qui s'origine, si l'on suit Claude Poliak, dans la « hantise de la prétention qui s'y trouve associée ", dans la « conscience de l'appartenance à un Nous », dans les suspicions également à l'égard de formes d'expression associées au féminin ${ }^{3}$. C'est ainsi que ce délégué CGT moque les manières de "pleureuses » qu'il prête à la plupart des porte-parole de sinistrés :

«Nous, on n'a pas des âmes de pleureuses, en tout cas moi, personnellement, je suis pas comme ça. En face par contre, y en a un paquet qui savent pleurer. ${ }^{4}$

1. Entretien, journaliste dans la presse quotidienne régionale, septembre 2002.

2. Ibid.

3. Claude Poliak, "Manières profanes de "parler de soi" ", Genèses, 47, 2002, p. 4-20.

4. Entretien, agent de sécurité à AZF, délégué CGT, avril 2003. 
Finalement, la nouvelle configuration contraindra malgré tout les syndicats d'AZF à un alignement a minima sur le registre victimaire d'expression des griefs, mais seulement à la marge et sous une forme braconnée. Un an après l'explosion, ils créent une association - AZF-Mémoire et solidarité - qui obtient - en tant qu' « association de défense de victimes d'accident collectif» - l'agrément du ministère de la Justice pour se porter partie civile lors du procès pénal. Bien sûr, le regroupement déroge aux canons de la forme d'action. Par exemple, si l'enjeu mémoriel est partagé avec de nombreuses associations de victimes, ce n'est pas le souvenir de l'accident qu'il s'agira d'entretenir, mais plutôt une histoire ouvrière antérieure à celui-ci. Mais l'endossement du statut juridique d'association de victimes ${ }^{1}$ pèse sur les manières de s'identifier et de se présenter publiquement. Les professionnels du droit associés à la cause des salariés d'AZF incitent ainsi les ouvriers de l'usine à l'adoption d'une qualification circonstancielle qui finit par être réappropriée.

«Pour les associations, les sinistrés, y avait qu'eux. Les sinistrés, c'était ceux de l'extérieur. C'est pour ça qu'on a été obligé de dire : "Les salariés sont les premiers parmi les sinistrés". Y en a $10 \%$ qui sont morts et y en a $100 \%$ qui ont perdu leur boulot. Alors merde, si ça c'est pas des sinistrés. Donc oui, c'est nous, les sinistrés. D'ailleurs, l'avocat nous l'a dit : "La demande d'agrément, quand même, vous pouvez la faire en tant que sinistrés, vous êtes tous des sinistrés". II nous a même dit que même les retraités étaient des sinistrés et c'est vrai qu'il y a eu, parmi les vieux, des mecs qui ont été traumatisés. $»^{2}$

\section{Être saisi par l'événement ou s'en saisir}

$\mathrm{L}$ a lecture "réactionnelle » de la genèse des mobilisations de "victimes » se nourrit finalement de sources - entretiens, matériaux de presse ou présentations publiques des associations - au prisme desquelles l'observateur risque de n'apercevoir que ce qu'on lui donne à voir : des "victimes" saisies par l'événement, plutôt que des acteurs concrets qui se saisissent de l'événement; des vocations militantes « révélées » par l'accident, plutôt que des carrières militantes au long cours que le contexte de catastrophe travaille et réoriente; des communautés d'accident liées par le partage indéfectible d'une expérience hors du commun, plutôt que des groupes enracinés qui se recomposent et renouent leurs alliances, au gré d'une configuration nouvelle. Cet article constitue, de ce point de vue, une invitation à l'exploration du "déjà-là ", même dans ces situations où semble régner le " jamais vu ». Dès lors que l'on rapproche la focale, les lois sociologiques du recrutement associatif conservent leur efficace, les supports organisationnels et les réseaux relationnels continuent d'affleurer, les dispositions militantes demeurent le tamis à travers lequel les expériences malheureuses sont filtrées pour devenir des engagements victimaires, etc. L'exemple d'AZF est ainsi sans doute un peu plus qu'idiosyncrasique. Que l'on songe au poids des groupes sexuels organisés dans le cas des rassemblements de victimes de viols ou du sida ${ }^{3}$; à celui des affinités confessionnelles dans les regroupements de victimes de sectes ${ }^{4}$; au rôle des réseaux militants de la lutte pour le droit au logement dans la création des associations de

\footnotetext{
1. Ce dernier est défini par l'article 2-15 du Code de procédure pénale.

2. Entretien, agent de maîtrise, président de l'association AZF-Mémoire et solidarité, avril 2003.

3. Christophe Broqua, Agir pour ne pas mourir. Act Up, les homosexuels et le sida, Paris, Presses de Sciences Po, 2005.

4. Étienne Ollion, "S'investir dans la lutte contre les sectes", mémoire pour la maîtrise de sociologie, Paris, Université Paris I-Panthéon Sorbonne, 2004.
} 
victimes d'incendies ou du saturnisme ${ }^{1}$; au concours du parti indépendantiste polynésien, de l'église protestante et des organisations pacifistes dans le mouvement des victimes des essais nucléaires ${ }^{2}$; à la forte contribution des réseaux combattants aux coalitions de victimes $\mathrm{du}$ "syndrome de la guerre du Golfe »" ; à la transformation de certaines sections syndicales en associations de victimes de l'amiante ${ }^{4}$; à l'inscription des mobilisations de sinistrés des marées noires dans les tissus locaux du militantisme associatif ${ }^{5}$; ou encore, aux multiples cercles de sociabilité (scoutisme, parents d'élèves, associations de loisirs) qui, bien souvent, nourrissent les mouvements ponctuels créés autour de la mort violente d'un enfant.

S'agit-il pour autant de réitérer à un énième plaidoyer pour le continuisme ? On s'exposerait alors à la critique du fixisme des sciences sociales que développent Alban Bensa et Éric Fassin.

«Nos disciplines préféreront le plus souvent montrer que l'événement n'en est pas un: la nouveauté n'est pas si nouvelle, le surgissement s'inscrit dans une perspective historique, une tradition culturelle, une logique sociale. Une fois encore, on s'emploie à réduire la surprise de l'événement: ce qui se passe était inscrit dans le passé, immédiat ou lointain - tout était déjà joué. ${ }^{6}$

Émettre l'hypothèse que l'événement n'impulse pas un renouvellement radical des raisons de se manifester (ou qu'il n'agit pas sur la nature de ces dernières qui deviendraient " émotionnelles») ne signifie pas pour autant le reléguer au statut d'épiphénomène. Nous avons en effet montré que l'occurrence de la catastrophe altère la structure des configurations dans lesquelles l'action collective se déploie et elle redéfinit les normes auxquelles doivent se conformer les acteurs. Elle modifie la hiérarchie relative des ressources à faire valoir (la démonétisation ponctuelle des capitaux politiques, la valorisation du statut de sinistré profane), elle pèse sur le choix des arènes à investir (la saillance conjoncturelles des arènes judiciaires et médiatiques), elle corrige les codes de la prise de parole (une contrainte de singularisation de l'expérience qui contraste avec les exigences habituelles du discours représentatif), etc. Or, c'est à l'aune de ces transformations qu'il convient d'envisager la recomposition des collectifs représentés (la conversion, par exemple, d'organisations de la gauche mouvementiste en "associations de sinistrés »), l'émergence de nouvelles identités revendiquées (celles de "victimes", de "familles endeuillées", de "sinistrés ») et l'entrée dans la lutte de nouveaux acteurs (une offre d'engagement conjoncturellement favorable aux carrières militantes de rattrapage). Aussi souscrirons-nous à la proposition de Jacques Lagroye qui, à la suite des travaux de Michel Dobry, affirmait que " [la crise] n'est pas une rupture : elle est la continuation d'un système de relations dans des conditions différentes » ${ }^{7}$.

\footnotetext{
1. Cécile Péchu, «Quand les exclus passent à l'action. La mobilisation des mal-logés », Politix, 34, 1996, p. 115-134, dont p. 125 et suiv.

2. Yannick Barthe, "Cause politique et "politique des causes". La mobilisation des vétérans des essais nucléaires français ", Politix, 91, 2010, p. 77-102.

3. Christine Abdelkrim-Delanne, Guerre du Golfe : la sale guerre propre, Paris, Cherche-Midi, 2001.

4. E. Henry, Amiante..., op. cit.

5. Xabier Itçaina, Julien Weisbein (dir.), Marées noires et politique. Gestion et contestation de la pollution du Prestige en France et en Espagne, Paris, L'Harmattan, 2011.

6. Alban Bensa, Éric Fassin, «Les sciences sociales face à l'événement», Terrain, 38, 2002, p. 5-20.

7. Jacques Lagroye, "Synthèse», dans Claude Gilbert (dir.), La catastrophe, l'élu et le préfet, Grenoble, Presses Universitaires de Grenoble, 1990, p. 209.
} 


\section{Stéphane Latté}

Maître de conférences en science politique à l'Université de Haute-Alsace, Stéphane Latté est membre du laboratoire PRISME-GSPE. Son doctorat (Prix AFSP/Mattéi Dogan, 2009) a notamment donné lieu à des articles consacrés à l'histoire des associations de victimes ("Victim Movements ", dans David A. Snow, Donatella Della Porta, Bert Klandermans, Doug McAdam (eds), The Blackwell Encyclopedia of Social and Political Movements, Chichester, Wiley Blackwell, 2012), à la sociologie des émotions et aux usages politiques du deuil (" "Vous ne respectez pas les morts d'AZF". Ordonner les émotions en situation commémorative", dans Sandrine Lefranc, Lilian Mathieu (dir.), Mobilisations de victimes, Rennes, Presses Universitaires de Rennes, 2009, p. 205-220), aux recours militants à la psychologie ("Enquête sur les usages sociaux du traumatisme », Politix, 73, 2006, p. 159-184). Parallèlement, il a également mené des enquêtes sur le recrutement politique local (« Cuisine et dépendance. Les logiques pratiques du recrutement politique ", Politix, 60, 2003, p. 55-81), la parité et la construction des identités genrées dans la vie politique municipale (avec Catherine Achin et alii., Sexes, genre et politique, Paris, Economica, 2007). Ses travaux portent sur la construction sociale de la catégorie de "victimes" et ses usages dans l'action collective (Université de Haute-Alsace, 16 rue de la Fonderie, 68093 Mulhouse cedex <stephane.latte@gmail.com>). 